\title{
Health procedures of the General Medical Council
}

\section{Sue Kesteven, Sheila Mann \& Andrew Sims}

The General Medical Council (GMC) relies upon consultant psychiatrists throughout the UK to assist in carrying out its twin tasks under the health procedures of protecting patients and guiding doctors. One of the reasons for the fitness of a doctor to be seriously impaired is the illness, usually mental illness, of that doctor, and frequently doctors do not realise the extent to which their ability to carry out their work has been affected.

Psychiatrists are often asked to help the GMC ensure that service for patients is safe and of an adequate standard by acting as supervisors, examiners and assessors. These tasks require additional knowledge, skills and judgement to those normally applied by psychiatrists. Those making referrals to and carrying out work for the GMC are often impatient with the protracted pace at which the organisation appears to work. It is slow but it is also thorough. Legal proof is often required and evidence must be collected that is beyond dispute.

Increasingly, the GMC is making its procedures transparent to try and remove the veil of mystery and fear which obscures its image, and is striving to make its mechanisms consistent in their application. The GMC aims to be fair in execution of its function by acting in the public interest, strictly within its statutory powers, and making decisions untainted with bias. This article describes the health procedures of the GMC, especially as applied to the work of consultant psychiatrists.

\section{Local intervention preferable}

Psychiatrists may be involved in caring for the health of their colleagues in many different ways, as, indeed, may specialists in other branches of medicine. However, given that most health conditions leading to concern over a doctors' fitness to practise are psychiatric, psychiatrists have a greater chance of being involved with the GMC's health procedures than their medical and surgical colleagues.

Often the first contact is an informal enquiry concerning the possible health problems of a colleague. In practice, procedures for dealing with sick doctors locally appear now to vary widely. In addition, there is often confusion as to the most appropriate way to raise concern over the psychiatric health of a colleague, and perhaps understandable reluctance to do so.

Psychiatrists consulted informally should bear in mind that the ideal solution for all is for a doctor to accept professional advice and treatment and to modify his or her practice in accordance with that advice. Thus, providing patient safety is not in doubt, the GMC's health procedures can be avoided. Efforts to persuade colleagues to accept local help and advice are always worthwhile. However, given that many mental health problems result in a lack of insight, and that most doctors are reluctant to accept that they might be suffering from a psychiatric problem, such local intervention may be unsuccessful.

It must be borne in mind that even if colleagues accept psychiatric advice, it is essential to make sure that they fully accept the dangers of practising if unwell. A reluctant acceptance of health problems must not hide a resolve to continue to practise as soon as possible, whatever the consequences. For example, a doctor may agree to early retirement on health grounds under pressure, including the 'threat' of referral to the GMC, but may then feel free to undertake other work, particularly in a locum capacity, before or

Sue Kesteven is Head of Health Section at the General Medical Council. Sheila Mann is Consultant/Senior Lecturer in Old Age with North East Essex Mental Health Services (Mental Health Administration, Clacton and District Hospital, Clacton-on-Sea, Essex CO15 1LH) and Andrew Sims is Professor of Psychiatry at the University of Leeds; both are members of the GMC involved in the Health Procedures. 
without full recovery. Psychiatrists, like other doctors, have an understandable reluctance to inform on colleagues and frequently feel acutely torn between the needs of doctor patients and the needs of the general public. It is always a tragedy if a patient's health is put in jeopardy because their doctor was unaware of or not prepared to admit to health problems of their own.

\section{Sick doctors and the GMC}

The GMC is the statutory body which regulates the medical profession in the interests of ensuring that those persons whose names appear in the Medical Register can safely be consulted by the public. To fulfil this function, the GMC has statutory procedures to deal with doctors' fitness to practise. These procedures are threefold: the disciplinary procedures, under which allegations of serious professional misconduct and convictions are considered; the performance procedures, introduced by the Medical (Professional Performance) Act 1995 and currently being established, which will deal with seriously deficient performance; and the health procedures, which are the subject of this article. A flow chart of the health procedures is set out in Fig. 1.

The health procedures are designed, first, to protect the public from doctors whose fitness to practise is seriously impaired on health grounds, and second, to assist the doctors concerned in following a programme of medical supervision and rehabilitation.

Definitions for each of the impairments to fitness to practise include the word 'seriously'. It is not the remit of the GMC to deal with every problem that arises with a doctor, however trivial. It is the GMC's concern to ensure that the registration of doctors who are liable to put patients at risk - for whatever reason - is restricted as necessary. In the context of the health procedures, this means that it is not for the GMC to deal with every doctor who is suffering from some form of illness. For example, it is assumed that a doctor who is prevented from working will seek appropriate medical advice, both about their condition and the extent to which they should limit their practice, if at all, on returning to work, in the interests of patients. The health procedures of the GMC are not intended to deal with such situations, or with doctors who take responsible action in relation to their health of their own volition. They are intended to protect patients from doctors who seek or continue to practise when they are not fit to do so and when their judgement is likely to be impaired. Serious impairment on health grounds means that a doctor's fitness to practise is so seriously impaired as to call into question the doctor's registration, in that his or her judgement or performance places patients or members of the public in jeopardy.

\section{Investigation}

The procedures are governed by statutory rules (The General Medical Council Health Committee (Procedure) Rules 1987). These require that, before action can be taken, the information alleging that the doctor is suffering from a serious health problem must be received in a proper form: that is, either from a "person acting in a public capacity" (as defined in the Rules), such as the Medical Director of a National Health Service trust or the Chairman of a local medical committee, or supported by a statutory declaration or affidavit.

All information received by the GMC that suggests that a doctor is suffering from a health problem, is considered by a medical member of the Council appointed to undertake the initial consideration of such cases, who is known as the Screener for Health. He or she will decide whether there is evidence of a health problem sufficiently serious to warrant initiating formal action under the health procedures. Further inquiries may be made, if necessary, but the Screener may conclude that there is insufficient evidence to justify taking the matter further. In reaching a decision, the Screener may take the view that local measures already in place to supervise and provide support for the doctor are sufficient to protect patients.

Invoking the health procedures is important from the point of view of protecting the public and can have potentially serious consequences for the doctor concerned, so the Screener will want to be satisfied that such action is justified and appropriate. He or she will be looking for firsthand evidence to substantiate the allegation that there is a health problem that is compromising patients' safety. For example, statements from colleagues who have witnessed the doctor under the influence of alcohol or drugs while on duty. The Screener cannot rely on hearsay or anecdotal evidence, and if the information received suggests that the doctor may be putting patients at risk, but there is no clear evidence of a health issue, the matter may more appropriately be dealt with under one of the other arms of the fitness to practise procedures. 


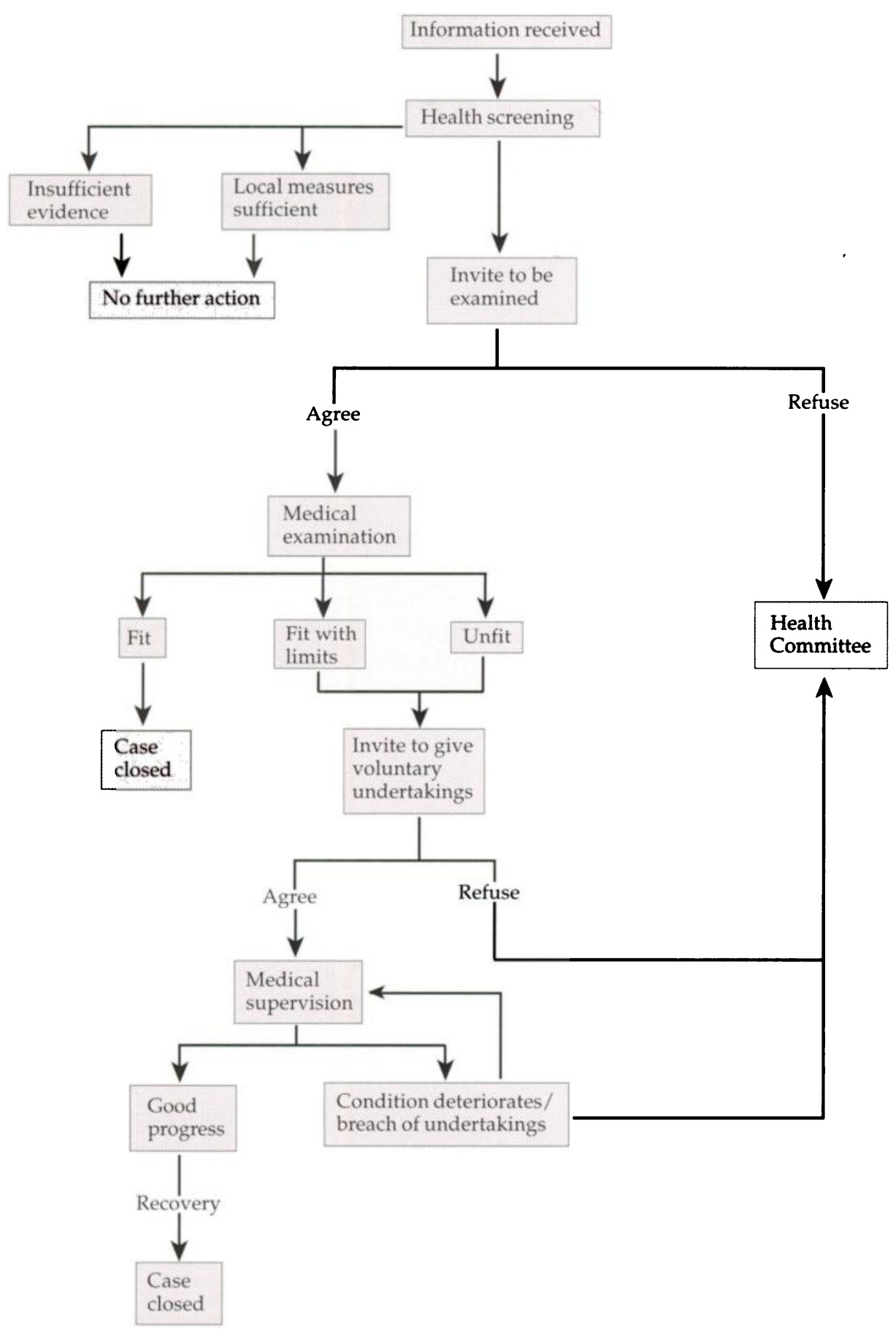

Iig. 1. Schematic diagram illustrating the General Medical Council's health procedure

\section{Medical examination}

If action is to be taken under the health procedures, then the first step is to invite the doctor to agree to medical examination by at least two examiners chosen by the Screener. Usually this will involve two psychiatric examinations but in some cases an examination by a physician or neurologist may also be necessary. Doctors are also offered the opportunity to nominate their own choice of examiner, although few take this up. In 1995, 46 doctors were invited to agree to medical examination, bringing the total of those asked since the health procedures began in 1980 to 606 . The majority of doctors considered under the health procedures are suffering from problems of addiction or other mental illness. The impairing conditions affecting those concerned are set out in Table 1. 
If the doctor agrees to medical examination, arrangements for the examinations are made and the examiners are asked to report to the GMC in terms of whether the doctor is fit to practise generally, without the need for medical supervision or any restriction on practice, or on a limited basis only, or not at all, and on how the doctor's case should be managed. On receipt of the medical reports, the Screener will take action based on what the examiners have recommended. If they conclude that the doctor is fully fit to practise, then the case will be closed. If, however, they conclude that the doctor is suffering from a health problem which impairs his or her fitness to practise, they will make recommendations for the doctor's medical supervision and treatment and to limit practice, perhaps even to the extent of proposing that the doctor should refrain from practice completely for a period.

The outcomes of the medical examinations of those examined under the procedures from 1980 to 1995 inclusive are set out in Table 2 .

\section{Medical supervision}

In cases where the doctor's fitness to practise is seriously impaired, the Screener will ask the doctor to give undertakings on a voluntary basis which reflect the advice the examiners have given. This normally entails placing the doctor under medical supervision with undertakings, which often include, for example, to abstain from alcohol and/ or refrain from self-medication.

If the doctor cooperates with this process, a system of monitoring is set up with a medical supervisor, nominated by the Screener, being asked to report regularly to the GMC on the doctor's progress. The medical supervisor will usually liaise with any consultant responsible for supervising the doctor's work or with other doctors working with him or her, as appropriate, as well as with the sick

Table 1. Number (\%) of cases considered under the health procedures, according to nature of the impairment, 1980-1995

Impairing condition

Alcohol only

Drugs only

$104(17)$

Psychiatric illness only

$147(24)$

Physical illness only

9 (1)

Illness involving two of the above

Illness involving three of the above

$155(26)$

$23(4)$

Total
Table 2. Outcome, in terms of fitness to practise, of GMC health procedure medical examinations, 1980-1995

FItness to practise $n(\%)$

Fit

Fit with limits

Unfit

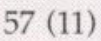

$337(66)$

$117(23)$

Total

$511(100)$

doctor's general practitioner and any other doctors involved in treatment.

In view of the fact that most doctors under the GMC's health procedures are suffering from relapsing conditions, supervision under the procedures may continue for a period of years. During that time, the doctor may be asked to give revised undertakings, if a relapse means that tighter restrictions are indicated, or where good progress is maintained and it is reasonable for restrictions to be relaxed. The aim is to reach a point where the doctor's condition has improved to such an extent that supervision and limitations on practice are no longer required to safeguard the public, and the doctor can be discharged from the health procedures. In 1995, 31 doctors were placed under medical supervision and 11 were discharged, having recovered to the point where they were considered fit to practise without the need for further supervision. This brought the total number of doctors under supervision to 147 by the end of the year.

The majority of those doctors who are referred to the GMC on health grounds cooperate with the procedures outlined above and are able to remain in practice, subject to whatever limitations are needed to ensure that they can practise safely.

\section{Health Committee}

A few doctors are either unable or unwilling to cooperate with the voluntary procedures, and this is where the Health Committee comes in. The Health Committee consists of nine members of the GMC - seven medical members and two lay members. The Committee sits in private and holds quasi-judicial hearings at which both the GMC and the doctor are entitled to be legally represented and oral evidence can be called if required. Generally, this is not necessary and the Committee relies on documentary evidence. The Committee is assisted at all hearings by a legal assessor, who advises on points of law, and by medical assessors. There are two categories of medical assessor. The 
first is chosen in relation to the nature of the condition alleged to impair the doctor's fitness to practise and thus is almost invariably a psychiatrist. It is the role of this assessor to advise the Committee on the significance of the medical evidence before it. It may on occasion be necessary to have more than one assessor to advise on the doctor's condition if, for example, it is complicated by a physical (e.g. neurological) problem. The second category of assessor is chosen from the same branch of medicine as the sick doctor to advise on the implications of the doctor's health problem as it is likely to affect the doctor's ability to practise in his or her chosen field of medicine.

Having considered all the evidence, the Committee must first determine whether the doctor's fitness to practise is seriously impaired. If the Committee decides that this is not so, the case will be concluded and that will be the end of the matter. If, however, the Committee does find serious impairment, it will go on to determine whether it is sufficient to impose conditions on the doctor's registration or whether the doctor's condition is such that there is no option but to suspend registration in order to protect the public. If conditions are imposed, they are likely to be similar to the undertakings given voluntarily by doctors under supervision under the health procedures, and will relate to the doctor's treatment and medical supervision and to restrictions on medical practice. Conditional registration is imposed for a finite period, at the end of which the case must be reviewed by the Health Committee. The same applies initially to suspension, but a new power has been introduced by the Medical (Professional Performance) Act 1995 which now enables the Committee to impose indefinite suspension, once a doctor's registration has been suspended for a total period of not less than two years.

A doctor can be referred to the Health Committee at any stage of the process. At the initial stage, if the doctor refuses to agree to be examined or fails to cooperate with arrangements for the examinations, he or she is liable to be referred to the Health Committee. In the absence of medical examinations, the information concerning the doctor's condition may be limited to the evidence which originally prompted a referral to the GMC, but the Committee is entitled to take account of the doctor's refusal or failure to be examined when reaching its decision on whether the doctor's fitness to practise is seriously impaired.

The next stage at which referral to the Health Committee becomes possible follows medical examination. If, having been examined, the doctor refuses to agree voluntarily to limit his or her practice to the extent recommended by the medical examiners, referral to the Health Committee is likely so that the Committee can decide the issue and, if necessary, impose conditions on the doctor's registration, or suspend it.

Finally, doctors who have cooperated with the health procedures and have been placed under medical supervision, having agreed to whatever limitations on practice have been deemed appropriate, may subsequently breach those undertakings, or their condition may subsequently deteriorate to the extent that they are no longer able to comply with them so that there is no option but to refer the doctor to the Health Committee, in order to ensure that the public are protected. By the end of 1995, there were 40 doctors under the jurisdiction of the Committee. Six new cases had been referred to the Committee during the course of the year and two doctors had been discharged, having been deemed fit to practise. Thus, even when a doctor's condition has been such that a referral to the Health Committee has been unavoidable, this does not mean that there is no hope of recovery and a number of doctors, albeit small, are discharged by the Committee each year.

\section{Psychiatrists as examiners, assessors and supervisors}

\section{Examination}

Psychiatrists are formally involved, as already indicated, in the Health Procedures as supervisors, examiners and assessors. The aim of a psychiatric examination is to achieve a comprehensive report on the diagnosis, possible causes, management and consequences of the doctor's illness, especially those effects which bear on the health and wellbeing of patients. Doctors undergoing examination are often ill and always stressed, and the Council tries to make sure that they do not have to travel too far. On the other hand, it is necessary to preserve impartiality and confidentiality and to make sure that examined and the examiner do not know each other professionally or socially.

Examiners are selected from a list of consultant psychiatrists nominated by the Royal College of Psychiatrists or the CCSC; new additions to this list are welcome. For each case the examiner is individually contacted by the GMC staff, usually by telephone, and has ample opportunity to clarify or to decline to undertake the examination if it is inappropriate or inconvenient. In view of the stress and distress to all, an early examination and report (within five weeks maximum) is desirable. If 
pressure of work or annual leave commitments make it difficult, it is better to decline at the start than to find oneself unable to comply with the deadline later on.

Doctors examining for the GMC are sent papers that include details of the circumstances giving rise to concern. This is confidential material, not to be revealed in detail to the doctor being examined. Usually the information is clear and unambiguous; sometimes it is not, and due weight has to be given to the various aspects. Examiners are asked to make contact, with the examined doctor's permission, with others who can shed light on the doctor's health and fitness to practise. This will usually include a close friend or relative, those with whom the doctor works, or his or her peers. Examiners have to bear in mind the ambivalence of many in providing information to the GMC, and the understandable wish of those close to a sick doctor to minimise (or occasionally to maximise) the degree of incapacity present. An ability to make judgements between different aspects of evidence, both written and in person, is essential.

Psychiatrists sometimes feel that because the majority of doctors referred to the GMC health procedures are suffering from problems of substance misuse (although by no means all, as is evident from Table 1), examining doctors need also to have a special knowledge of substance misuse. This is far from the truth. What is required is a comprehensive report taking into account all factors. Although an initial supposition from the papers may be that drug or alcohol misuse is the major problem, this may not prove to be the case.

Tact and consideration must be used in arranging the appointment with the doctor and his or her friends or colleagues. Even though the examination may take place at some distance from the doctor's own practice, doctors are extremely sensitive to being recognised and gossiped about and it is usually more appropriate to arrange a specific appointment than to include examinations in the course of a clinic or other regular commitment. Many doctors being examined are not working at the time of examination, which makes day-time appointments feasible in many instances, but by no means all, and the offer of a late afternoon or early evening appointment is often much appreciated.

Examiners are asked to carry out any physical examination, tests or investigations thought necessary to establish the diagnosis, management and likely outcome. However, since it is both upsetting for the doctor and unnecessarily expensive, examiners are asked to arrange for one set of investigations with their fellow examiner, although it is expected that the reports will be independent and there will be no exchange of views between examiners, tempting though this may be.

Where an examination for the GMC differs from other psychiatric examinations for reports is in the requirement to assess fitness to practise. It is necessary to look at the effect of the illness on medical judgement (both at the time and bearing in mind that the impairment may be episodic or recurring) and on the doctor's ability to carry out their work appropriately in the future. Some conditions manifest themselves with a mixed medical and psychiatric picture, and not all impairing conditions are psychiatric, so when appropriate, an examination by a physician or neurologist or another specialist will be arranged. Staff of the Health Section of the GMC are willing to advise; if in doubt as to whether further investigation or examination is appropriate, it is always wise to discuss this with them.

Having dispatched the report (and received the fee), examiners usually hear no more until a brief note informing them in strict confidence of the outcome of the case. Although almost always the examined doctors accept the findings of the examiners, occasionally they do not and the matter then becomes one for the Health Committee, at which point the examiner may be called to appear at its hearing. On occasion, examiners are asked regularly to re-examine a doctor whose case is being considered by the Health Committee, and this has a particular interest since the progress (or otherwise) of the doctor can be followed.

\section{Assessment}

Assessors for the Health Committee, mostly drawn from those who have previously examined or supervised, sit with the Health Committee usually for one day at a time, and advise on all aspects of the medical evidence. As indicated, there are two assessors and one is almost always a psychiatrist. To be an effective assessor it is necessary to be able to weigh up the comparative importance of written reports and oral opinions before and at the time of the hearing. This requires considerable concentration as well as an ability to sum up evidence and to be able to present it succinctly and clearly to those who, as is the case in the majority of Health Committee members, have no specialist psychiatric knowledge. The aim is to make clear the points which Health Committee members need to consider, while avoiding giving a didactic lecture on psychiatry. The assessor must also advise on 
Box 1. Summary

The GMC has a statutory duty to regulate the practice of the medical profession in order to protect patients and guide doctors.

On the basis of a preliminary screening, health procedures may be set up, which first require the doctor to be medically examined.

Either because local measures have proved effective or because there seems to be no impairment of fitness to practise, the case may be closed.

The case may be managed using voluntary undertakings of the doctor in informal procedures or, more rarely, may require referral to the Health Committee.

Almost all cases of severe impairment of fitness to practise on grounds of health are 'psychiatric': diagnoses of alcohol misuse are most common.

Psychiatrists are involved in assisting the GMC in the capacity of examiners, assessors and supervisors.

Health procedures of the GMC are directed specifically at protecting patients and guiding doctors, with that order of priority.

whether the evidence suggests that the doctor is seriously impaired - not always an easy task given the episodic and relapsing nature of some conditions, but nevertheless essential as the Health Committee can only act when impairment is deemed serious.

\section{Supervision}

A further way in which psychiatrists may become involved in GMC procedures is perhaps the most enduring and important - as supervisors for doctors who have agreed to supervision under the Health Procedures. Supervisors may be responsible for the psychiatric treatment of the sick doctor (indeed may have treated him or her before referral to the GMC) or may be invited to supervise a doctor who is already receiving treatment from other colleagues. Very often, one of the psychiatrists providing the initial examination is invited to supervise the doctor. This has obvious advantages but is not always acceptable to the doctor concerned.

To supervise well is an art. Many doctors are resentful and suspicious of a supervisor, at least initially. However, unless a relationship of trust can be established, supervision may be difficult for the supervisor and not helpful for the doctor or the GMC. Supervisors need to use their judgement as to how often they should see a doctor and how often they should contact others such as relatives, friends and those with whom the doctor works, if this is a requirement of the supervision arrangements. Supervisors are often given very consider- able powers, for example to request a doctor to cease work immediately if the supervisor is sufficiently concerned about fitness to practise. They will also in many instances be in touch with a sick doctor's colleagues and able to enhance or diminish the doctor's chance of reintegration into normal professional life. Doctors under supervision vary in their attitudes and willingness to comply with the GMC procedures, necessitating discernment on the part of the supervisor who may need to decide whether compliance is wholehearted or grudging. Supervisors are asked to make regular reports at intervals determined by the GMC and dependent on the doctors' health and general situation. Supervising a sick doctor who improves, accepts advice, resumes work gradually and ultimately returns fully to practise is extremely rewarding, but not a task to be undertaken lightly.

Finally, we have to accept that we are all vulnerable to psychiatric disorders, however unwilling we may be to contemplate this. Experience of working with sick doctors and the GMC emphasises the importance of seeking help early, accepting professional advice and thereby avoiding the formality and stress of a contact with the Council as patient, rather than physician.

\section{Further reading}

Connell, P. (1991) Ten years of the Health Procedures. In GMC Annual Report 1990, pp. 9-11. London: GMC.

Kessel, N. (1996) Ill doctors. Psychiatric Bulletin, 20, 577-579. 


\section{Multiple choice questions}

1. At hearings of the Health Committee:

a two psychiatrists act as assessors only when the sick doctor works in psychiatry

b the press are usually in attendance

c three lay members of the GMC must be present

$\mathrm{d}$ both the GMC and the sick doctor may be legally represented

e if fitness to practise is impaired, but not seriously so, the case is concluded without further action.

2. Medical examination for the Fitness to practise Division of the GMC:

a can be carried out by any psychiatrist who holds a Certificate of Completion of Specialist Training

$b$ requires the production of a written report which will be shown to the sick doctor

c will normally recommend undertakings that affect working conditions

$\mathrm{d}$ indicates more problems of drug misuse than alcohol misuse among doctors

e is usually carried out in a different health region than that in which the doctor practises.
3. Medical supervision of a sick doctor by the GMC:

a may be initiated when fitness to practise is only moderately impaired

b usually involves liaison with the practitioner supervising the sick doctor's work

$c$ is the most frequent outcome following medical examination

$\mathrm{d}$ is frustrated in most cases by the doctor's non-compliance

e can only be carried out by approved examiners.

$\begin{array}{clll}\text { MCQ answers } & & & \\ \text { 1 } & \text { 2 } & 3 \\ \text { a T } & \text { a F } & \text { a F } \\ \text { b F } & \text { b T } & \text { b T } \\ \text { c F } & \text { c T } & \text { c T } \\ \text { d T } & \text { d F } & \text { d F } \\ \text { e T } & \text { e F } & \text { e F }\end{array}$

MCQ answers 